\title{
Use of Generalized Fluid System Simulation Program (GFSSP) for Teaching and Performing Senior Design Projects at the Educational Institutions
}

\author{
A. K. Majumdar and A. Hedayat \\ Propulsion Systems Department \\ Marshall Space Flight Center, MSFC, AL 35812
}

\begin{abstract}
This paper describes the experience of the authors in using the Generalized Fluid System Simulation Program (GFSSP) in teaching Design of Thermal Systems class at University of Alabama in Huntsville. GFSSP is a finite volume based thermo-fluid system network analysis code, developed at NASA/Marshall Space Flight Center, and is extensively used in NASA, Department of Defense, and aerospace industries for propulsion system design, analysis, and performance evaluation. The educational version of GFSSP is freely available to all US higher education institutions. The main purpose of the paper is to illustrate the utilization of this user-friendly code for the thermal systems design and fluid engineering courses and to encourage the instructors to utilize the code for the class assignments as well as senior design projects.
\end{abstract}

The need for a generalized computer program for thermofluid analysis in a flow network has been felt for a long time in aerospace industries. Designers of thermofluid systems often need to know pressures, temperatures, flow rates, concentrations, and heat transfer rates at different parts of a flow circuit for steady state or transient conditions. Such applications occur in propulsion systems for tank pressurization, internal flow analysis of rocket engine turbopumps, chilldown of cryogenic tanks and transfer lines, and many other applications of gas-liquid systems involving fluid transients and conjugate heat and mass transfer. Computer resource requirements to perform time-dependent, three-dimensional Navier-Stokes computational fluid dynamic (CFD) analysis of such systems are prohibitive and therefore are not practical.

Available commercial codes are generally suitable for steady state, single-phase incompressible flow. Because of the proprietary nature of such codes, it is not possible to extend their capability to satisfy the above-mentioned needs. Therefore, the Generalized Fluid System Simulation Program $\left(\right.$ GFSSP $^{1}$ ) has been developed at NASA Marshall Space Flight Center (MSFC) as a general fluid flow system solver capable of handling phase changes, compressibility, mixture thermodynamics and transient operations. It also includes the capability to model external body forces such as gravity and centrifugal effects in a complex flow network. The objectives of GFSSP development are: a) to develop a robust and efficient numerical algorithm to solve a system of equations describing a flow network containing phase changes, mixing, and rotation; and b) to implement the algorithm in a structured, easy-to-use computer program.

The analysis of thermofluid dynamics in a complex network requires resolution of the system into fluid nodes and branches, and solid nodes and conductors as shown in Figure 
1. Figure 1 shows a schematic and GFSSP flow circuit of a counter-flow heat exchanger. Hot nitrogen gas is flowing through a pipe, colder nitrogen is flowing counter to the hot stream in the annulus pipe and heat transfer occurs through metal tubes. The problem considered is to calculate flowrates and temperature distributions in both streams.

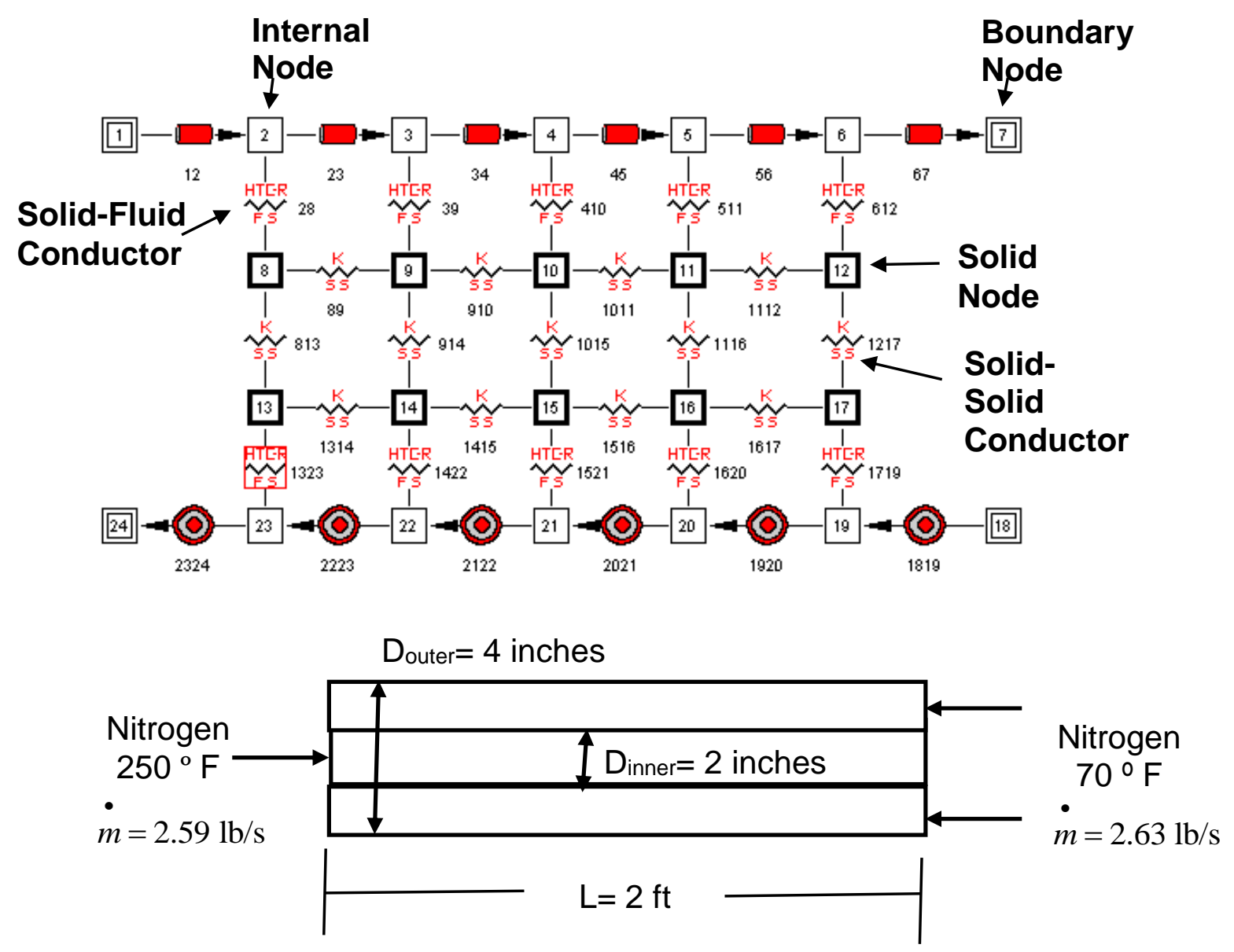

Figure 1. A Typical Flow Network consists of Fluid Node, Solid Node, Flow Branches and Conductors

GFSSP has a unique data structure, as shown in Figure 2, that allows constructing all possible arrangements of a flow network with no limit on the number of elements. The elements of a flow network are boundary nodes where pressure and temperature are specified, internal nodes where pressure and temperature are calculated, and branches where flowrates are calculated. For conjugate heat transfer problems, there are three additional elements: solid node, ambient node, and conductor. The solid and fluid nodes are connected with solid-fluid conductors. 


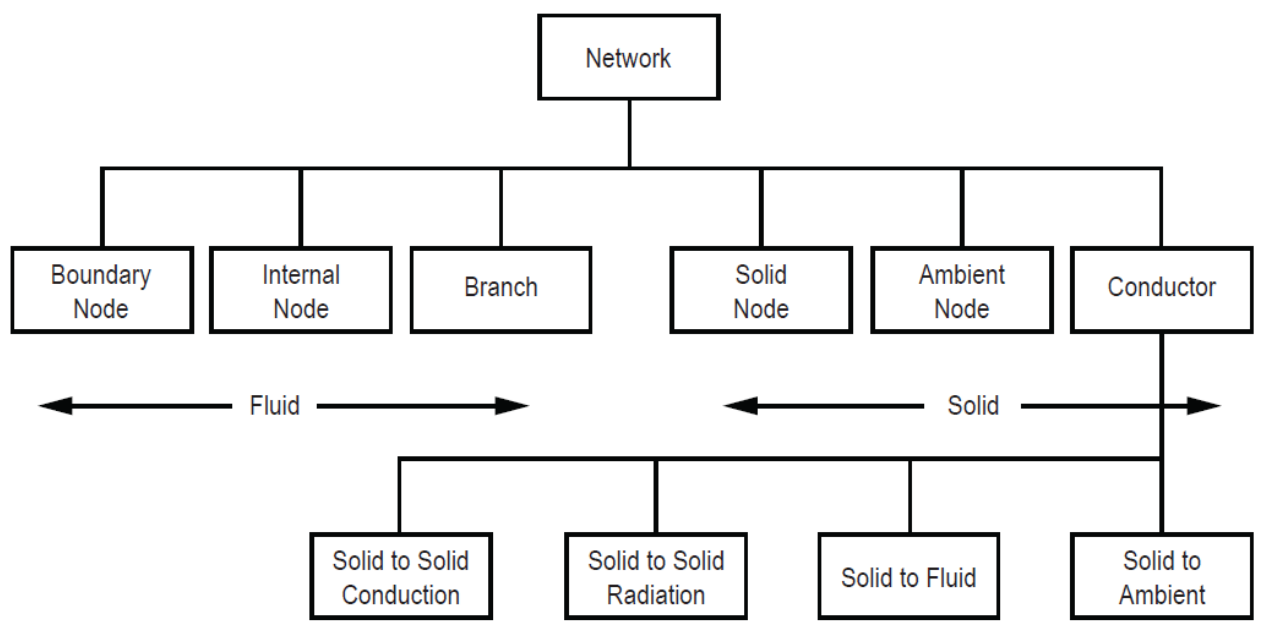

Figure 2. Data structure of the fluid-solid network has six major elements.

GFSSP solves the conservation equations of mass and energy, and equation of state in internal nodes to calculate pressure, temperature and resident mass. The momentum conservation equation is solved in branches to calculate flowrate. It also solves for energy conservation equations to calculate temperatures of solid nodes. The equations are coupled and nonlinear; therefore, they are solved by an iterative numerical scheme. GFSSP employs a unique numerical scheme known as simultaneous adjustment with successive substitution (SASS), which is a combination of Newton-Raphson and successive substitution methods. The mass and momentum conservation equations and the equation of state are solved by the Newton-Raphson method while the conservation of energy and species are solved by the successive substitution method.

GFSSP is linked with two thermodynamic property programs, GASP ${ }^{2}$ and $\mathrm{WASP}^{3}$ and $\mathrm{GASPAK}^{4}$, that provide thermodynamic and thermophysical properties of selected fluids. Both programs cover a range of pressure and temperature that allows fluid properties to be evaluated for liquid, liquid-vapor (saturation), and vapor region. GASP and WASP provide properties of 12 fluids. GASPAK includes a library of 36 fluids.

GFSSP has three major parts. The first part is the graphical user interface (GUI), visual thermofluid analyzer of systems and components (VTASC). VTASC allows users to create a flow circuit by a 'point and click' paradigm. It creates the GFSSP input file after the completion of the model building process. GFSSP's GUI provides the users a platform to build and run their models. It also allows post-processing of results. The network flow circuit is first built using three basic elements: boundary node, internal node, and branch.

Mathematical formulation of GFSSP is based on a Newtonian, nonreacting and onedimensional flow in the flow circuit. The flow can be steady or unsteady, laminar or 
turbulent, incompressible or compressible, with or without heat transfer, phase change, mixing, and rotation.

During the class periods, the students learned to use the program through lectures and hands-on tutorial problems. They were assigned homework problems to use the code in developing models of flow network consisting of components such as pipe, valve, bend, nozzle, and pump with given characteristics and flow distribution in manifold. Finally, they were given a project assignment of designing a chilled water air-conditioning system in a multistoried building where they used the code to select the pump and to size the pipes to ensure the desired flow distribution in all stories. The final paper will describe in more details the tutorials, homework problems, and design project assignment with sample flow models developed by the students.

\section{References}

1. A.K. Majumdar, A.C. LeClair, R. Moore, P.A. Schallhorn, Generalized Fluid System Simulation Program, Version 6.0, NASA/TM-2013-217492 (October 2013) <https://gfssp.msfc.nasa.gov>.

2. Hendricks, R.C.; Baron, A.K.; and Peller, I.C.: "GASP - A Computer Code for Calculating the Thermodynamic and Transport Properties for Ten Fluids: Parahydrogen, Helium, Neon, Methane, Nitrogen, Carbon Monoxide, Oxygen, Fluorine, Argon, and Carbon Dioxide," NASA TN D-7808, NASA Lewis Research Center, Cleveland, OH, February 1975.

3. Hendricks, R.C.; Peller, I.C.; and Baron, A.K., "WASP - A Flexible Fortran IV Computer Code for Calculating Water and Steam Properties," NASA TN D-7391, NASA Lewis Research Center Cleveland, OH, November 1973.

4. User's Guide to GASPAK, Version 3.20, Cryodata, Inc., November 1994. 